\title{
TERAPIA OCUPACIONAL COMUNITARIA Y REHABILITACION BASADA EN LA COMUNIDAD: HACIA UNA INCLUSION SOCIOCOMUNITARIA
}

\author{
COMMUNITY OCCUPATIONAL THERAPY AND COMMUNITY BASED \\ REHABILITATION: TOWARDS INCLUSION SOCIOCOMMUNITY
}

Juan Pino $M^{1}$, Margareth Ceballos $C^{2}$

\section{RESUMEN}

El presente manuscrito es un ejercicio teórico/práctico realizado para apoyar una propuesta disciplinar emergente. El objetivo es visibilizar la necesidad de realizar intervenciones que apunten a lograr una inclusión sociocomunitaria Se elaboró luego de revisar y analizar los resultados y conclusiones de una investigación de magister y la práctica profesional del autor y la autora en la estrategia de Rehabilitación Basada en la Comunidad en las comunas de Hualpén y Talcahuano, en la región del Biobío. A partir de ahí, se articularon constructos epistemológicos para una propuesta metodológica de intervención de Terapia Ocupacional Comunitaria que permita comprometerse con la realidad y las necesidades ocupacionales de las personas de nuestro espacio regional geopolítico. El trabajo se estructuró en torno a aproximaciones conceptuales de Terapia Ocupacional Comunitaria y luego una lectura aplicada en la estrategia de Rehabilitación Basada en la Comunidad.

\footnotetext{
${ }^{1}$ Terapeuta Ocupacional, Licenciado en Ciencias de la Ocupación Humana, Magíster en Psicología Comunitaria. Académico Universidad Nacional Andrés Bello. Código postal 4260000, fono: 91620282 juanpino.to@gmail.com

2 Terapeuta Ocupacional, Licenciada en Terapia Ocupacional, Magíster en Terapia Ocupacional mención psicosocial (C) Universidad Nacional Andrés Bello. Código postal 4260000, fono: 51790685 magit_ap@hotmail.com
} 
Palabras claves: Terapia Ocupacional Comunitaria, Inclusión Sociocomunitaria, Rehabilitación Basada en la Comunidad.

\begin{abstract}
This manuscript is a theoretical / practical exercise conducted to support a proposal emerging discipline. The aim is to demonstrate the need for interventions aimed at achieving sociocommunitarian inclusion is then prepared to review and analyze the results and conclusions of research master's and professional practice of the author and the author's strategy Based Rehabilitation Community in the communes of Hualpén and Talcahuano, in the Biobio region . From there, articulated epistemological constructs a methodology for intervention allowing Community Occupational Therapy compromise with reality and occupational needs of the people in our regional geopolitical space. The work is structured around conceptual approaches Community Occupational Therapy and then a reading strategy applied in the Community Based Rehabilitation.
\end{abstract}

Keywords: Community Occupational Therapy, Socio-Community Inclusion, Community Based Rehabilitation. 


\section{Introducción}

El presente trabajo tiene el objetivo de generar reflexiones y problematizaciones en torno a la Terapia Ocupacional Comunitaria (TOC) con el fin de atreverse a presentar una propuesta metodológica para la intervención comunitaria desde la Terapia Ocupacional (T.O). En ésta se articulan y destacan los conceptos centrales que deberían aparecer para comprender y dimensionar una intervención integrativa con enfoque comunitario. Esta dimensión y comprensión de la T.O proviene de lo que plantea Morrison, Olivares y Vidal (2011) como paradigma social de la ocupación.

La propuesta proviene de la inquietud de la autora y el autor en sistematizar experiencias y saberes conceptuales que posibiliten contar con mayores herramientas para el trabajo en comunidad y así enriquecer la propuesta tradicional y clásica del trabajo de T.O en los escenarios sociales y contemporáneos a los cuales se vincula. A su vez, quiere fortalecer y aportar a lo planteado por Oyarzun, Zolezzi, Núñez y Palacios (2009) en "Hacia la construcción de prácticas comunitarias de terapeutas ocupacionales de Chile", donde refieren que existe una dominancia de enfoques y modelos anglosajones, que separan los aspectos individuales y comunitarios. Por último, contar con mayores conocimientos teóricos y metodológicos para la inserción de Terapeutas Ocupacionales Comunitarios altamente demandados en los servicios públicos y sociales del Estado.

Como premisa central, en torno a la que se desarrolla este escrito, planteamos que el ser es “ontológicamente comunitario y ocupacional". Por tanto, nos hacemos parte o inclinamos por proposiciones tales como "al existir persona existe ocupación" entendiendo ésta última como "la síntesis de nosotros, es lo que nos hace ser nos-otros” (Ramírez, Shliebener, 2014). Bajo esta óptica planteamos que la persona está anclada al mundo de la vida, que es el mundo de lo cotidiano, de la experiencia, del sentido común, del mundo comunitario, preocupación principal para esta dimensión de la Terapia Ocupacional. En este mundo primordialmente se tejen los sentidos y significados de las ocupaciones, se construye, re-construye y de-construye la vida, es donde la o el sujeto se valida, se 
reconoce, adquiere identidad, pertenencia, donde se juega el devenir de su existencia, es en el mundo comunitario el espacio donde se resuelven la mayoría de las problemáticas cotidianas, es por esto que creemos que para lograr la inclusión, las políticas públicas, las instituciones y disciplinas que las operacionalizan deben tener como finalidad la inclusión "sociocomunitaria" de las personas. En base a esto sugerimos una metodología o un posible modelo (flexible, discutible y abierta a transformaciones) que se aleje de las conceptualizaciones intelectualistas y abstractas que están imponiéndose con fuerza en la disciplina en este último tiempo. Nuestra preocupación es por la acción y la praxis que enriquece la teoría, desde la cual se incluye y relaciona conceptos desde el enfoque comunitario y Terapia Ocupacional.

Se utiliza como referencia preferentemente el trabajo desarrollado por el profesor Víctor Martínez, Psicólogo Comunitario de la Universidad de Chile, quien ha estado debatiendo fuertemente en las Ciencias Sociales y las Políticas Públicas la importancia de la "Comunidad" como unidad de análisis, intervención y transformación. Así mismo, las fuentes epistemológicas que emplea la Terapia Ocupacional Comunitaria provienen de una raíz latinoamericana destacando en este punto a la Psicología de la liberación, Educación Popular, el trabajo de Maritza Montero, Orlando Fals Borda, Ignacio Martin Baró, entre otras.

Para este trabajo, consideramos la comunidad parafraseando a Martínez (2014) como una caja negra a la cual todos se refieren, se sabe que existe, que está allá afuera, se conocen sus efectos, pero es difícil de encontrar, de objetivar, de manipular, sin embargo, está dada por personas que comparten un "estar en común" en un mundo compartido, una comunidad no es un sujeto colectivo, es una "comunidad de los otros" que se construye sobre el principio de alteridad. La comunidad sitúa a las y los individuos en lo social, asignándole identidad, validación, reconocimiento, pertenencia, territorios, lengua y cultura.

Consideramos a la comunidad como un ente nunca neutro, más bien lo concebimos en su dimensión crítica, entrecruzado por las lógicas de poder y las fuerzas culturales hegemónicas, 
pensamos en este artículo como un instrumento de lucha y transformación para T.O comunitarios críticos, con posición política, cuyo sustento valórico y ético lo constituyen los derechos humanos. Comprometidos con la transformación y el cambio social que favorezca a comunidades y personas a las que abocamos nuestras acciones profesionalmente diarias (Guajardo, 2014). Apostamos por la interculturalidad, la descolonización del saber y el respeto por la diferencia, la autonomía, la interdependencia y la liberación.

\section{Aproximaciones conceptuales de Terapia Ocupacional Comunitaria}

Figura 1: aproximaciones conceptuales de Terapia Ocupacional Comunitaria

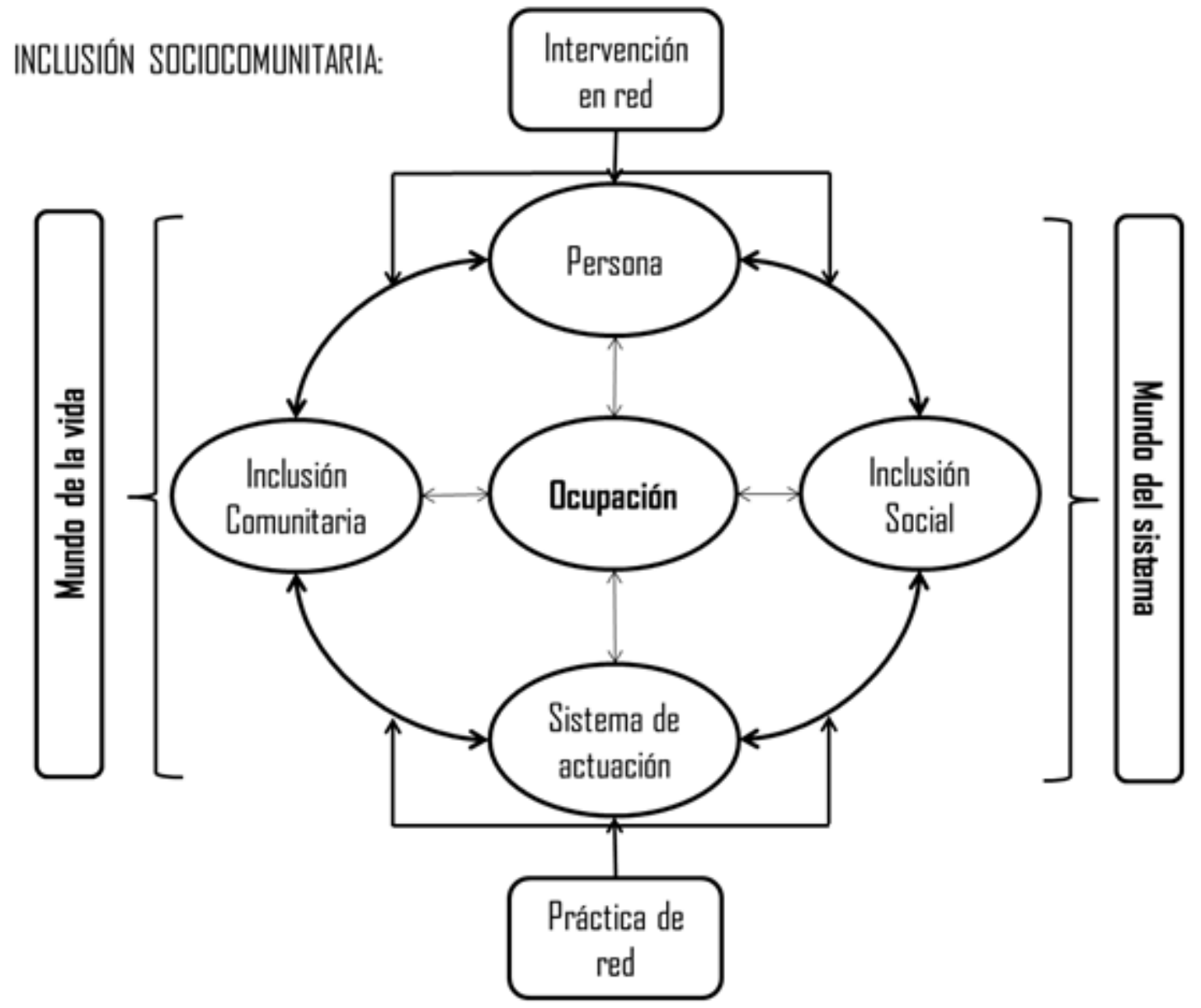

Revista Chilena de Terapia Ocupacional 5 
La persona: es un ser ontológicamente comunitario y ocupacional (Ceballos, Pino y Sepúlveda, 2015). Siempre está en relación a otras/otros, la persona está anclada al mundo de la vida y al mundo del sistema de una manera dialógica, irreversible, histórica, situada. Ésta no vive sola, sino que vive en comunidades, esas comunidades generan ocupaciones con sentidos colectivos, las personas producen sentidos de pertenencia, identificaciones en curso como lo plantea De Sousa (2009). Por tanto, cuando nos referimos a las personas, nos referimos a la ocupación humana, son inseparables, es su esencia, su naturaleza, es constituyente del sujeto, como proceso constructor de sujetos y subjetividades (Trujillo, Sanabria, Carrizosa, Parra, Rubio, Uribe et al. 2011). Es nuestro foco de toda propuesta interventiva.

La Ocupación Humana: es el eje central de la T.O, por medio de éstas las y los sujetos "desarrollan sus potenciales biológicos, individuales y socioculturales para hacerse sujeto, gestor y constructor de su propia historia" (Trujillo et al. 2011, p. 50). Las ocupaciones tienen siempre lugar en un contexto determinado, definido por dimensiones espaciales y temporales: todas las ocupaciones son prácticas situadas, localizadas, territorializadas, y se dan en un aquí y ahora específicos, concreto e irreversible. El contexto es siempre dinámico ya que está siendo construido, reconstruido y deconstruido constantemente. La ocupación humana no es un quehacer aislado, es relacional, comunica, "somos lo que hacemos" y a través del hacer construimos y de-construimos el mundo.

Desde una perspectiva crítica Ramírez y Shliebener $(2014$, p. 8) "La ocupación como potencial humano va mutando a través de las tensiones y luchas sociales, los cambios de paradigmas, el desarrollo de las fuerzas productivas. Las posturas triunfantes en la hegemonía histórica y social de ciertos sectores van determinando o imponiendo cuales son las ocupaciones válidas para cada tipo de sociedad y cuáles son los válidos para desempeñarla".

Mundo de la Vida: se referirse a la experiencia cotidiana y natural de los individuos en tanto sujetos desde su posibilidad de acción, reconociéndoles la posibilidad de posicionarse como actores de cambio de su propio entorno. Salas (2006, p. 74) lo plantea como "si este mundo de la vida refiere 
al mundo de la experiencia cotidiana cabe asumir que el sujeto es un actor que vivencia significativamente este mundo. En segundo lugar, está llamado a actuar en él y sobre él. En tercer lugar, el vivir implicaría necesariamente actividad en el mundo. El mundo de la vida cotidiana, en síntesis, sería el entorno vital mismo desde el que se sitúa el sujeto humano, y cabe entender su relación con otros y con la naturaleza". El concepto mundo de la vida refiere a una propuesta epistémica para la compresión del mundo del sentido común.

Mundo del Sistema: es el mundo institucional tradicional (dominante e instaurado en Chile), es el espacio del trabajo, de las relaciones más estructuradas, jerarquizadas e instrumentalizadas. Está construido bajo una lógica digital, lineal, racional, con una distribución organigrámica del poder, una alta concentración de la toma de decisiones y un alto nivel de formalización de los procedimientos internos; las relaciones con las personas son de tipo secundario, de roles predefinidos, quedan relegadas a roles pasivos, como pacientes, usuarios, beneficiarios, clientes, importa más el rol asignado que la personalidad de la o el sujeto" (Martínez, 2006, p. 61). Se rige por la lógica de la eficiencia y eficacia, de la optimización y de la racionalización. La institución depende de un presupuesto, funciona sobre la base de reglas explícitas y orientadas hacia el logro de una misión, de objetivos y metas. En base a esta epistemología buscan construir el mundo al que se dirigen.

Sistemas de Actuación: son los sistemas espaciales y simbólicos en los cuales se estructuran los vínculos entre personas, se instalan en territorios en un tiempo determinado (para transformarlo en un decurso histórico, en pasado, presente, futuro), es fijación ordenadora desde el sentido de objetos y de personas en alteridad. Se liga a las características de la estructura en las que las personas se incluyen o excluyen, es campo activo, entramado afectivo y emocional, marco conversacional, es molde perceptivo, es un sistema de habitudes, tiene lugar en el proceso de personalización de los individuos que lo componen. Los sistemas de actuación pueden estar inmersos en el mundo de la vida (comunidad) como en el mundo del sistema (instituciones). Son las disposiciones y capacidades de las personas al interior de las comunidades e instituciones (Martínez, 2014).

Inclusión Comunitaria: es la inserción activa o participación de las personas en el mundo de la vida, que es la expresión de la lógica de los sistemas naturales e íntimos, que les proporciona: vínculos con los demás y las cesas en un campo-especial temporal concreto, histórice, sittrado, deRevista Chilena de Terapia Ocupacional 7 
lugares, contextos, situaciones. Un campo práctico de convivencia con los demás, donde vivencia y experimenta lo común, donde participa en una comunidad; un campo concreto de rutinas, de usos y costumbres, saberes y valores. Proporciona pertenencia, identidad, protección, seguridad, afecto, estima, valoración, reconocimiento, apoyo, compenetración personal. Sistemas de posibilidades para sus procesos de personalización y autoconfiguración (Martínez, 2014).

Inclusión social: es la inserción, participación y acceso de las personas en un sistema de actuación de formalidad institucional (mundo del sistema). Se constituye como un mundo más impersonal y artificial, donde lo relevante es la conexión operativa de las personas con lo institucional. En este campo se ejerce la ciudadanía, ya que se sustenta en la perspectiva de derecho tradicional. Es en definitiva, el pleno uso que hace una persona de los derechos de la sociedad en que participa. Por lo tanto podemos identificar como expresiones de inclusión social, lo referente al acceso y participación en los ámbitos educaciones, laborales, recreaciones, culturales, de salud, direccionados por la instituciones del Estado.

Intervención en Red: es un proceso profesionalizante directo, se incluyen todas aquellas conversaciones-acciones que implican un contacto directo de un equipo especializado o de la o el Terapeuta Ocupacional en la intervención con el sujeto-foco personalizado (persona, pareja, familia, grupo, comunidad). Tal como su nombre lo indica, la estrategia pasa por una intervención en la red del sujeto-foco con el propósito de desarrollarla operativamente en función de los problemas y necesidades de éste, de reconstruirla o sustituirla en caso de disfuncionamiento y de construirla cuando no existe. Las principales funciones de la intervención en red son las siguientes: proporciona un apoyo afectivo, contribuye a co-construir el problema con la familia, proporciona una información esencial a la comprensión del problema (Martínez, 2006).

Práctica de Red: es un tipo de intervención técnica, que se expresa en una secuencia programada de conversación-acción que tiene lugar entre un equipo profesional y un conjunto de actores sociales los cuales pueden tener un carácter institucional o comunitario o ambos con el propósito de: detectar 
redes sociales ya existentes en un territorio determinado (por ejemplo una comuna) y en relación a una temática específicas (por ejemplo, prevención del consumo de drogas, del maltrato infantil, de la violencia intrafamiliar, etc.); fortalecer y optimizar el funcionamiento de las redes sociales existentes; crear contextos favorables a la emergencia de nuevas redes sociales abiertas. La principal herramienta de la práctica de red es su dimensión dialógica, materializada en la conversación, implica relación directa, cara a cara, con los actores correspondientes. En el interior de la conversación se desarrollan vínculos funcionales y operativos para resolver problemas, se espera que se cree la mayor cantidad de sistemas de vínculos (Martínez, 2006).

\section{Análisis y reflexiones de las aproximaciones conceptuales de Terapia Ocupacional Comunitaria en Rehabilitación Basada en la Comunidad en Chile}

Figura 2: aplicación de las aproximaciones conceptuales de Terapia Ocupacional Comunitaria en Rehabilitación Basada en la Comunidad en Chile

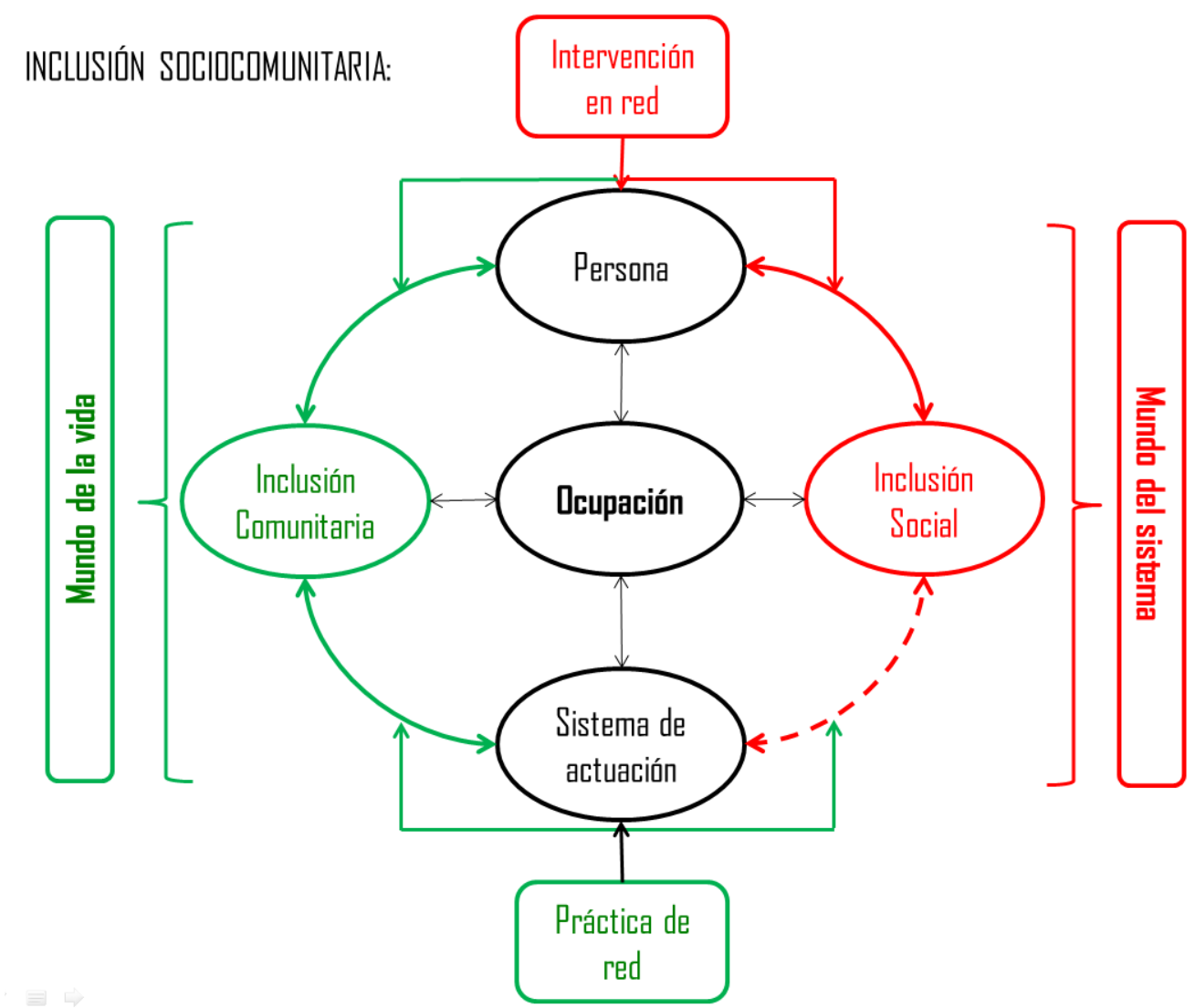


Esta experiencia y reflexiones están basadas en el trabajo práctico y sistematización de la Terapeuta Margaret Ceballos y en los hallazgos encontrados en la investigación realizada por el Terapeuta Juan Pino a la estrategia de Rehabilitación Basada en la Comunidad (RBC) en la Región del Biobío de Chile, específicamente en las comunas de Talcahuano y Hualpén durante los años 2012-2014.

El esquema representa el estado del arte que se logró identificar en términos de lo que se plantea como aproximaciones conceptuales de la Terapia Ocupacional Comunitaria, el color rojo representa el principal desarrollo y orientación de la situación actual de la estrategia y el color verde representa la deuda que está pendiente impulsar desde el posicionamiento que tiene el manuscrito.

A raíz de lo anterior se pudo concluir, según la perspectiva de las personas en situación de discapacidad (PsD) pertenecientes a la estrategia de RBC, que la inclusión sociocomunitaria ha sido fragmentada, destacando más bien espacios de "integración/inclusión social", dominados principalmente por el acceso a la esfera médica, respuesta principal del mundo del sistema, lo que a su vez, es concordante con un paradigma "Rehabilitador" de la Discapacidad, que desde su filosofía considera que las causas que originan la discapacidad son científicas, derivadas en limitaciones individuales de las personas (Pino, 2014). Bajo esta óptica, las personas en situación de discapacidad son consideradas necesarias y útiles para la sociedad, pero siempre en la medida en que sean rehabilitadas a través de intervenciones en red preferentemente, de ahí la relevancia de los Centros Comunitarios de Rehabilitación (CCR) propuestos por la estrategia en Chile (Palacios, 2008).

Es por ello, que el fin primordial que se persigue desde este modelo es normalizar a las personas con discapacidad, aunque ello implique la desaparición o el ocultamiento de la diferencia que la misma discapacidad representa. El problema cardinal pasa a ser, entonces, la persona, con sus diversidades y dificultades, a quien es imprescindible rehabilitar psíquica, física, mental o sensorialmente. 
Desde un análisis con el marco conceptual de la T.O comunitaria se puede concluir que la experiencia de la RBC en Talcahuano y Hualpén ha evidenciado un gran problema en el componente del mundo de la vida, nudo crítico de la arquitectura de la estrategia en Chile, dificultando el logro de los objetivos propuestos en materia de inclusión sociocomunitaria. Esto, dada la poca capacidad de fortalecer las esferas naturales, ecológicas y cotidianas de las personas en situación de discapacidad. Muy por el contrario, teniendo una preocupación desde lo institucional y mundo del sistema, el modelo propone como fundacional el trabajo principalmente con sistemas naturales, es decir, con sistemas no creados por los programas o proyectos de intervención, articulando así una mirada que pone en tensión el caso de los CCR como espacio angular y principal de la estrategia chilena de RBC en la actualidad.

Consideramos que lo anterior se expresa en la convivencia cotidiana de las personas en situación de discapacidad pertenecientes a la RBC. Ésta se encuentra muy condicionada por el mundo compartido, sus experiencias y oportunidades; estas personas, de un mismo mundo (discapacidad) se relacionan habitualmente con las mismas personas (personas en situación de discapacidad, en Centros Comunitarios de Rehabilitación), los mismos objetos (implementos terapéuticos, talleres, ayudas técnicas), son manipuladas aproximadamente de la misma manera, experimentan las mismas vivencias y necesidades (necesidades principalmente médicas, de rehabilitación y apoyo), se regulan por el mismo cuerpo de reglas, opinan de manera semejante, comparten valores semejantes, comparten las mismas expectativas, manejan los mismos modelos de nivel y calidad de vida.

De aquí que las personas en situación de discapacidad de la RBC consideran, en su mayoría, que sus dificultades, necesidades y problemas, tienen relación con un problema médico; esto le da un carácter identitario a su existencia, a su ocupación y su comunidad, cosa que se acentúa al estar y recibir principalmente una relación vinculante con el sistema de salud, de ahí la necesidad de incorporar nuevos sistemas de actuación, vinculado a sus espacios comunitarios y naturales, también favorecer a través de acceso a otros escenarios sociales e institucionales que permitan hacer efectivos su variedad de derechos. 
Desde las aproximaciones conceptuales de la T.O comunitaria se plantea una metodología donde exista un equilibrio de articulaciones, esta mirada se refiere a la interpenetración, comunicación y diálogo entre el mundo de la vida/mundo del sistema, en esa interfaz se produce la inclusión sociocomunitaria, esto significaría que las personas en situación de discapacidad desde su posición situacional al interior de estos sistemas, contribuyen a construir los contextos en los cuales son construidos como sujetos sociales "incluidos" o "excluidos" sociocomunitariamente.

Nuestro posicionamiento debe sugerir una comprensión distinta de la y él sujeto. Referimos entonces que el ser es "ontológicamente (un ser) comunitario y ocupacional", diremos que éste no se puede separar de estas dos naturalezas, sino que se necesitan, son interdependientes, están interactuando y dialogando constantemente, aún más, son condición sí, sólo sí, una de la otra. Al estar inmersas, una en la otra, reconocemos que si hablamos del "ser" necesariamente hablamos de "ocupación y comunidad", no hay ser sin ocupación ni comunidad, la existencia de "comunidad y ocupación" hablan de la existencia de un/una ser (por sí mimas), si tenemos seres singulares entonces tenemos comunidades y ocupaciones, si tenemos ocupaciones tenemos comunidades conformadas por seres singulares, todo esto para dejar expuesto que el ser singular es ontológicamente "comunitario y ocupacional".

La ocupación es un proceso constituyente del ser humano, por tanto, al comprender las interacciones que se dan entre ambos mundos, es posible comprender a las entidades mismas, instalando así la relación persona-ocupación. Respecto a esto hablamos de un/una ser singular que constituye su singularidad, sus identificaciones en un mundo con otros/otras y que en estas relaciones dan sentido y significado a sus praxis, reconocemos en las ocupaciones un componente histórico, situado, que influencia y valida ciertas prácticas, a través de éstas el ser construye, reconstruye y deconstruye su contexto siempre en relación a otro u otra. Es relevante pensar en términos de las ocupaciones que desarrollan las personas pertenecientes a la estrategia de la RBC en Chile, debido a que preferentemente se desarrollan al interior de los mundos institucionales. 
Por tanto, cuando trabajamos en la estrategia de RBC debemos considerar que estamos frente a un/una ser singular, que se articula con otras/otros en un estar-en-común (comunidad), espacio donde genera sus identificaciones, diálogos, visiones de mundo, una/un ser singular autónomo, interdependiente, con un bagaje histórico, con distintos saberes que deben ser validados, un sujeto que no es una persona en situación de discapacidad (PsD), ni "enfermo con algún apellido", sino que es un ser singular parte del entramado social. Un ser que expresa su singularidad, que transforma y es transformado a partir de las articulaciones, entendidas como lo que da sentido y significado a su hacer. Debemos relacionarnos de manera tal que permitamos la expresión del ser singular que es también la comunidad -en tanto que es constituyente de esta y expresa en su voz singular, las voces de las y los distintos participantes de ésta- ya no como agentes externos, interventores sino inmiscuirnos en el mundo particular de las y los sujetos, esto supone relaciones horizontales, de diálogo, respeto y validación de la y el otro como experto de su situación, por tanto posicionarnos como parte (par-te, como par y parte) de la comunidad. (Nancy, 2010).

Es vital que se fortalezca el modelo de inclusión sociocomunitaria en la estrategia de la RBC, con nuevos sistemas de vínculos, con nuevas membranas comunicacionales, preferentemente que se fortalezca los ámbitos de la inclusión comunitaria y como rol primordial de las y los profesionales el desarrollo de la práctica de red con la finalidad de activar nuevos sistemas de actuaciones de las personas en situación de discapacidad, identificando, reforzando y construyendo mayores convergencias discursivas, alcanzando mayores consensos entre el mundo institucional y el mundo de la vida.

\section{Referencias Bibliográficas}

Ceballos, M., Pino, J., Sepulveda, R. (2015). Terapia Ocupacional Comunitaria Critica: Dialogos y Reflexiones para iniciar una propuesta colectiva. Revista Gallega de Terapia Ocupacional.

Guajardo, A. (2014). Chile Terapia Ocupacional Apuntes para historia inconclusa. En V. Dos Santos, A. Donatti, Cuestiones Contemporáneas de Terapia Ocupacional en América del Sur. (pp. 51-72). Curitiba: CRV. 
Martínez, V. (2006). El enfoque comunitario: el desafio de incorporar a las comunidades en las intervenciones sociales. Santiago de Chile: Universidad de Chile.

Martínez, V. (2014). Modelo de inclusión sociocomunitaria, documento en borrador. Universidad de Chile.

Morrison, R., Olivares, D., Vidal, D. (2011). La filosofía de la Ocupación Humana y el paradigma social de la Ocupación. Algunas reflexiones y propuestas sobre epistemologías actuales en Terapia Ocupacional y Ciencias de la Ocupación. Revista Chilena de Terapia Ocupacional, 11(2), 102-119.

Nancy, J. (2000). La Comunidad Inoperante. Santiago de Chile: Arces-LOM.

Oyarzun, N., Zolezzi, R., Núñez, J., Palacios, M. (2009). Hacia la construcción de las prácticas comunitarias de terapeutas ocupacionales en Chile, desde una mirada socio histórica, desde 1972 hasta la actualidad. Revista Chilena de Terapia Ocupacional, 0 (9), 149-165.

Palacios, A. (2008). El modelo social de discapacidad: orígenes, caracterización y plasmación en la Convención Internacional sobre los Derechos de las Personas con Discapacidad. Madrid: Grupo Editorial Cinca.

Pino, J. (2014). Nada de Nosotros, sin Nosotros: "Experiencias de rehabilitacion basada en la comunidad, VIII región, Chile". En A. Guajardo, E. Recabarren, V. Parraguez, Rehabilitacion de Base Comunitaria. (pp. 163-182). Santiago de Chile.

Ramírez, R., Shliebener, M. (2014). Manifiesto Latinoamericano de Terapia Ocupacional y Ocupación. Revista Gallega de Terapia Ocupacional, 11(19), 1-18.

Salas, R. (2006). El mundo de la vida y la fenomenología sociológica de Schütz: apuntes para una filosofía de la experiencia. Revista de Filosofía, 15, 167- 199. 
De Sousa, B. (2009). Una epistemología del Sur. Buenos Aires: siglo veintiuno ediciones.

Trujillo, A., Sanabria, L., Carrizosa L., Parra E., Rubio S., Uribe J., et al (2011). Ocupación: sentido, realización y libertad, diálogos ocupacionales en torno al sujeto, la sociedad y el medio ambiente. Bogotá: Universidad Nacional de Colombia. 